\title{
Comparing Bug Finding Tools with Reviews and Tests $^{\star}$
}

\author{
Stefan Wagner ${ }^{1}$, Jan Jürjens ${ }^{1}$, Claudia Koller ${ }^{1}$, and Peter Trischberger ${ }^{2}$ \\ 1 Institut für Informatik, \\ Technische Universität München, \\ Boltzmannstr. 3, D-85748 Garching, Germany \\ ${ }^{2} \mathrm{O}_{2}$ Germany, Georg-Brauchle-Ring 23-25, \\ D-80992 Munich, Germany
}

\begin{abstract}
Bug finding tools can find defects in software source code using an automated static analysis. This automation may be able to reduce the time spent for other testing and review activities. For this we need to have a clear understanding of how the defects found by bug finding tools relate to the defects found by other techniques. This paper describes a case study using several projects mainly from an industrial environment that were used to analyse the interrelationships. The main finding is that the bug finding tools predominantly find different defects than testing but a subset of defects found by reviews. However, the types that can be detected are analysed more thoroughly. Therefore, a combination is most advisable if the high number of false positives of the tools can be tolerated.
\end{abstract}

\section{Introduction}

Software failures can have enormous consequences in terms of threatening peoples lives as well as economic loss because various critical systems rely on software. Furthermore, software becomes increasingly complex, which makes the prevention of failures even more difficult. However, software quality assurance accounts already for around $50 \%$ of the development time 13. Therefore it is important to improve defect-detection techniques as well as reduce their costs. Automation can be an option in that direction. For example, automated test-case generation based on executable models is also under investigation as a possibility to make testing more efficient [16.

Extensive research has been done on finding defects in code by automated static analysis using tools called bug finding tools, e.g. 1, 1, 8. Although the topic is subject of ongoing investigations, there are only few studies about how these tools relate among themselves and to other established defect-detection techniques such as testing or reviews.

\footnotetext{
* This research was supported in part by the Deutsche Forschungsgemeinschaft (DFG) within the project InTime.
} 
We will now discuss the problem situation in more detail. We briefly define the terms we use in the following: Failures are a perceived deviation of the output values from the expected values whereas faults are the cause of failures in code or other documents. Both are also referred to as defects. We mainly use defect in our analyses also if there are no failures involved as with defects related to maintenance only.

Problem. We address the question of how automated static analysis using bug finding tools relates to other types of defect-detection techniques and if it is thereby possible to reduce the effort for defect-detection using such tools. In detail, this amounts to three questions.

1. Which types and classes of defects are found by different techniques?

2. Is there any overlap of the found defects?

3. How large is the ratio of false positives from the tools?

Results. The main findings are summarised in the following.

1. Bug finding tools detect a subset of the defect types that can be found by a review.

2. The types of defects that can be found by the tools can be analysed more thoroughly, that is, the tools are better regarding the bug patterns they are programmed for.

3. Dynamic tests find completely different defects than bug finding tools.

4. Bug finding tools have a significant ratio of false positives.

5. The bug finding tools show very different results in different projects.

Consequences. The results have four major implications.

1. Dynamic tests or reviews cannot be substituted by bug finding tools because they find significantly more and different defect types.

2. Bug finding tools can be a good pre-stage to a review because some of the defects do not have to be manually detected. A possibility would be to mark problematic code so that it cannot be overlooked in the review.

3. Bug finding tools can only provide a significant reduction in the effort necessary for defect-detection if their false positives ratios can be reduced. From our case studies, we find the current ratios to be not yet completely acceptable.

4. The tools have to be more tolerant regarding the programming style and design to provide more uniform results in different projects.

Experimental Setup. Five industrial projects and one development project from a university environment were selected which are either already in use or in the final testing phase. We evaluated several bug finding tools and chose three representatives that were usable for the distributed web systems under consideration. The bug finding tools and dynamic tests were used on all projects. A review was only possible for a single project. The warnings issued from the tools were analysed with experienced developers to classify them as true and false positives. All defects that were found were classified regarding their severity and defect types. The comparison was done based on this classification. 
Contribution. We are not aware of studies that compare the defects found by bug finding tools with the defects found by other techniques, in particular not of any based on several, mainly industrial, projects. A main contribution is also a thorough analysis of the ratio of false positives of the bug finding tools as this is a significant factor in the usability of these tools.

Organisation. Sec. 2 gives an overview of bug finding tools in general (Sec. 2.1) and the three tools that were used in the projects (Sec. 2.2). The projects are described in Sec. 3 with general characteristics in Sec. 3.1] and specific descriptions in Sec. 3.2 The approach for the comparison of the techniques can be found in Sec. 4 with a general discussion in Sec. 4.1, the defect classification in Sec. 4.2 and the introduction of the defect types in Sec.4.3. The analysis of the study is described in Sec. 5 with the comparison among the bug finding tools in Sec. 5.1 bug finding tools versus reviews in Sec. 5.2. bug finding tools versus testing in Sec. 5.3, and the defect removal efficiensies in Sec. 5.4. We discuss our findings in Sec. 6] and describe related work in Sec. 7. Finally, we conclude in Sec. 8 and sketch intended future work in Sec. 9 .

\section{Bug Finding Tools}

This section provides an introduction to bug finding tools in general and describes briefly the three tools that were used in the case study.

\section{$2.1 \quad$ Basics}

Bug finding tools are a class of programs that aim to find defects in code by static analysis similarly to a compiler. The results of such a tool are, however, not always real defects but can be seen as a warning that a piece of code is critical in some way. There are various techniques to identify such critical code pieces. The most common one is to define typical bug patterns that are derived from experience and published common pitfalls in a certain programming language. Furthermore, coding guidelines and standards can be checked to allow a better readability. Also, more sophisticated analysis techniques based on the dataflow and controlflow are used. Finally, additional annotations in the code are introduced by some tools [7] to allow an extended static checking and a combination with model checking.

\subsection{Analysed Tools}

The three bug finding tools that we used for the comparison are described in the following. We only take tools into account that analyse Java programs because the projects we investigated, as described below, are all written in that language. All three tools are published under an open source license. We used these three tools as representatives for tools that mainly use bug patterns, coding standards, and dataflow analysis, respectively. We deliberately ignored tools that need annotations in the code because they have quite different characteristics. 
FindBugs. The tool FindBugs was developed at the University of Maryland and can detect potentially problematic code fragments by using a list of bug patterns. It can find faults such as dereferencing null-pointers or unused variables. To some extent, it also uses dataflow analysis for this. It analyses the software using the bytecode in contrast to the tools described in the following. The tool is described in detail in [8]. We used the Version 0.8.1 in our study.

$P M D$. This tool [15] concentrates on the source code and is therefore especially suitable to enforce coding standards. It finds, for example, empty try/catch blocks, overly complex expressions, and classes with high cyclomatic complexity. It can be customised by using XPath expressions on the parser tree. The version 1.8 was used.

QJ Pro. The third tool used is described in [17] and analyses also the source code. It supports over 200 rules including ignored return values, too long variable names, or a disproportion between code and commentary lines. It is also possible to define additional rules. Furthermore, checks based on code metrics can be used. The possibility to use various filters is especially helpful in this tool. We evaluated version 2.1 in this study.

\section{$3 \quad$ Projects}

We want to give a quick overview of the five projects we analysed to evaluate and compare bug finding tools with other defect-detection techniques.

\section{$3.1 \quad$ General}

All but one of the projects chosen are development projects from the telecommunications company $\mathrm{O}_{2}$ Germany for backend systems with various development efforts and sizes. One project was done by students at the Technische Universität München. All these projects have in common that they were developed using the Java programming language and have an interface to a relational database system. The $\mathrm{O}_{2}$ projects furthermore can be classified as web information systems as they all use HTML and web browsers as their user interface.

\subsection{Analysed Projects}

The projects are described in more detail in [12]. For confidentiality reasons, we use the symbolic names A through $\mathrm{D}$ for the industrial projects.

Project $A$. This is an online shop that can be used by customers to buy products and also make mobile phone contracts. It includes complex workflows depending on the various options in such contracts. The software has been in use for six months. It consists of 1066 Java classes that consist of over 58 KLOC (kilo lines of code). 
Project $B$. The software allows the user to pay goods bought over the Internet using a mobile phone. The payment is added to the mobile bill. For this, the client sends the mobile number to the shop and receives a transaction number (TAN) via short message service (SMS). This TAN is used to authenticate the user and authorises the shop to bill the user. The software has not been put into operation at the time of the study. Software B has 215 Java classes with over 24 $\mathrm{KLOC}$ in total.

Project $C$. This is a web-based frontend for managing a system that is used to convert protocol files between different formats. The tool analysed only interacts with a database that holds administration information for that system. The software was three months in use at the time it was analysed. It consists of over 3 KLOC Java and JSP code.

Project $D$. The client data of $\mathrm{O}_{2}$ is managed in the system we call $D$. It is a J2EE application with 572 classes, over 34 KLOC and interfaces to various other systems of $\mathrm{O}_{2}$.

$E s t A$. The only non-industrial software that we used in this case study is EstA. It is an editor for structuring textual requirements developed during a practical course at the Technische Universität München. It is a Java-based software using a relational database management system. The tool has not been extensively used so far. It has 28 Java classes with over 4 KLOC.

\section{Approach}

In this section, the approach of the case study is described. We start with the general description and explain the defect classification and defect types that are used in the analysis.

\subsection{General}

We use the software of the five projects introduced in Sec. 3 to analyse the interrelations between the defects found by bug finding tools, reviews, and tests. For this, we applied each of these techniques to each software as far as possible. While a review was only made on project $\mathrm{C}$, black-box as well as white-box tests were done on all projects. We ran the bug finding tools with special care to be able to compare the tools as well. To have a better possibility for comparison with the other techniques, we also checked each warning from the bug finding tools if it is a real defect in the code or not. This was done by an inspection of the corresponding code parts together with experienced developers. The usage of the techniques was completely independent, that is, the testing and the review was not guided by results from the bug finding tools.

The external validity is limited in this case study. Although we mostly considered commercially developed software that is in actual use, we only analysed 
five systems. For better results more experiments are necessary. Furthermore, the tests on the more mature systems, i.e. the ones that are already in use, did not reveal many faults. This can also limit the validity. Moreover, the data from only one review is not representative but can only give a first indication. Finally, we only analysed three bug finding tools, and these are still under development. The results might be different if other tools would have been used.

In the following we call all the warnings that are generated by the bug finding tools positives. True positives are warnings that are actually confirmed as defects in the code, false positives are wrong identifications of problems.

\subsection{Defect Categorisation}

For the comparison, we use a five step categorisation of the defects using their severity. Hence, the categorisation is based on the effects of the defects rather than their cause or type of occurrence in the code. We use a standard categorisation for severity that is slightly adapted to the defects found in the projects. Defects in category 1 are the severest, the ones in category 5 have the lowest severity. The categories are:

1. Defects that lead to a crash of the application. These are the most severe defects that stop the whole application from reacting to any user input.

2. Defects that cause a logical failure. This category consists of all defects that cause a logical failure of the application but do not crash it, for example a wrong result value.

3. Defects with insufficient error handling. Defects in this category are only minor and do not crash the application or result in logical failures, but are not handled properly.

4. Defects that violate the principles of structured programming. These are defects that normally do not impact the software but could result in performance bottlenecks etc.

5. Defects that reduce the maintainability of the code. This category contains all defects that only affect the readability or changeability of the software.

This classification helps us (1) to compare the various defect-detection techniques based on the severity of the defects they find and (2) analyse the types of defects that they find.

\subsection{Defect Types}

Additionally to the defect classification we use defect types. That means that the same or very similar defects are grouped together for an easier analysis. This is not based on any standard types such as [2,4,9] but was defined specifically for the applications.

The defect types that we use for the bug finding tools can be seen as a unification of the warning types that the tools are able to generate. Examples for defect types are "Stream is not closed" or "Input is not checked for special characters". 


\section{$5 \quad$ Analysis}

This section presents the results of the case study and possible interpretations. At first, the bug finding tools are compared among each other, then the tools are compared with reviews, and finally with dynamic tests.

\subsection{Bug Finding Tools}

We want to start with comparing the three bug finding tools described in Sec. 2 among themselves. The tools were used with each system described above.

Data. Tab. 1) shows the defect types with their categories and the corresponding positives found by each tool over all systems analysed. The number before the slash denotes the number of true positives, the number after the slash the number of all positives.

Observations and Interpretations. Most of the true positives can be assigned to the category Maintainability of the code. It is noticeable that the different tools predominantly find different positives. Only a single defect type was found from all tools, four types from two tools each.

Considering the categories, FindBugs finds in the different systems positives from all categories and PMD only from the categories Failure of the application,

Table 1. Summary of the defect types found by the bug finding tools

\begin{tabular}{|l|r|r|r|r|}
\hline Defect Type & Category & FindBugs & PMD & QJ Pro \\
\hline Database connection is not closed & 1 & $8 / 54$ & $8 / 8$ & $0 / 0$ \\
Return value of function ignored & 2 & $4 / 4$ & $0 / 0$ & $4 / 693$ \\
Exception caught but not handled & 3 & $4 / 45$ & $29 / 217$ & $30 / 212$ \\
Null-pointer exception not handled & 3 & $8 / 108$ & $0 / 0$ & $0 / 0$ \\
Returning null instead of array & 3 & $2 / 2$ & $0 / 0$ & $0 / 0$ \\
Stream is not closed & 4 & $12 / 13$ & $0 / 0$ & $0 / 0$ \\
Concatenating string with + in loop & 4 & $20 / 20$ & $0 / 0$ & $0 / 0$ \\
Used "==" instead of "equals" & 4 & $0 / 1$ & $0 / 0$ & $0 / 29$ \\
Variable initialised but not read & 5 & $103 / 103$ & $0 / 0$ & $0 / 0$ \\
Variable initialised but not used & 5 & $7 / 7$ & $152 / 152$ & $0 / 0$ \\
Needless if-clause & 5 & $0 / 0$ & $16 / 16$ & $0 / 0$ \\
Multiple functions with same name & 5 & $22 / 22$ & $0 / 0$ & $0 / 0$ \\
Needless semicolon & 5 & $0 / 0$ & $10 / 10$ & $0 / 0$ \\
Local variable not used & 5 & $0 / 0$ & $144 / 144$ & $0 / 0$ \\
Parameter not used & 5 & $0 / 0$ & $32 / 32$ & $0 / 0$ \\
Private method not used & 5 & $17 / 17$ & $17 / 17$ & $0 / 0$ \\
Empty finally block & 5 & $0 / 0$ & $1 / 1$ & $0 / 0$ \\
Needless comparison with null & 5 & $1 / 1$ & $0 / 0$ & $0 / 0$ \\
Uninitialised variable in constructor & 5 & $1 / 1$ & $0 / 0$ & $0 / 0$ \\
For- instead of simple while loop & 5 & $0 / 0$ & $2 / 2$ & $0 / 0$ \\
\hline
\end{tabular}




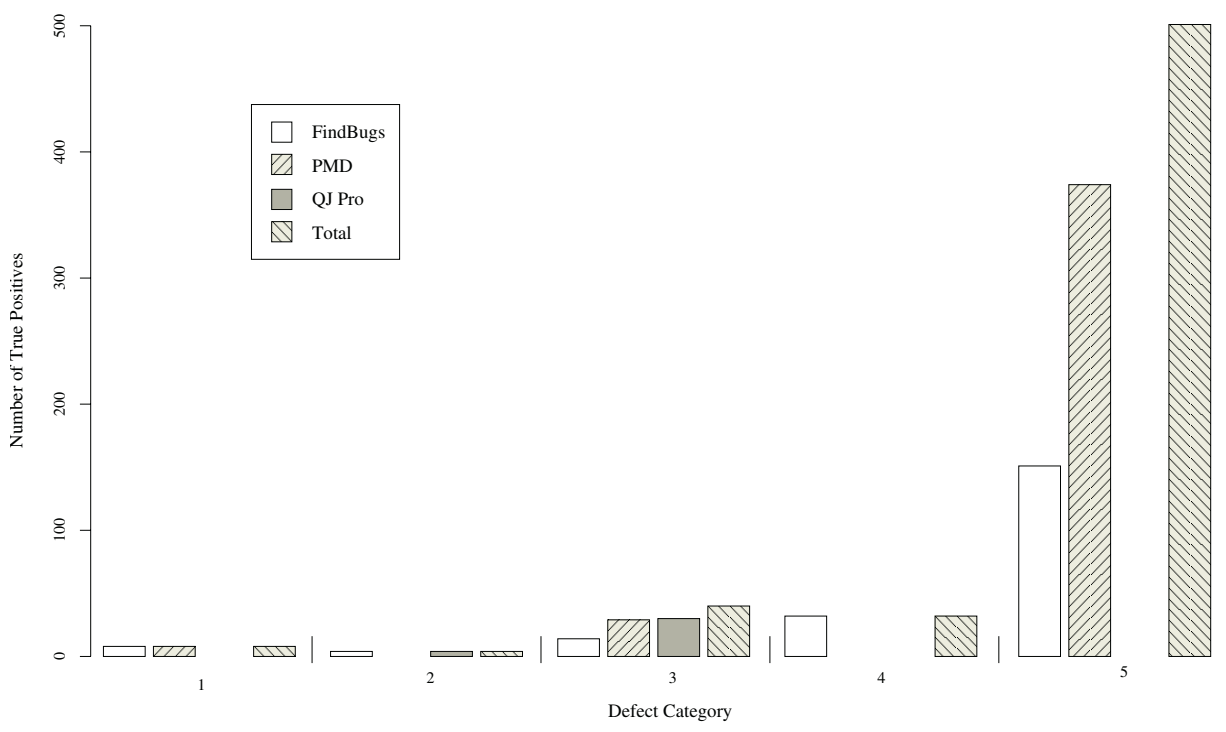

Fig. 1. A graphical comparison of the number of true positives found by each tool and in total

Insufficient error handling, and Maintainability of the code. QJ Pro only reveals positives from the categories Logical failure of the application, Insufficient error handling, and Violation of structured programming. The number of faults found in each category from each tool is graphically illustrated in Fig. 1]. Also the number of types of defects varies from tool to tool. FindBugs detects defects of 13 different types, PMD of 10 types, and QJ Pro only of 4 types.

The accuracy of the tools is also diverse. We use the defect type "Exception is caught but not handled" that can be found by all three tools as an example. While FindBugs only finds 4 true positives, PMD reveals 29 and QJ Pro even 30. For this, the result from QJ Pro contains the true positives from PMD which in turn contain the ones from FindBugs. A reason for this is that QJ Pro is also able to recognize a single semicolon as an non-existent error handling, whereas the other two interpret that as a proper handling. This defect type is also representative in the way that FindBugs finds the least true positives. This may be the case because it uses the compiled class-files while PMD and QJ Pro analyse the source code.

A further difference between the tools is the ratio of true positives to all positives. PMD and FindBugs have a higher accuracy in indicating real defects than QJ Pro. Tab. 2 lists the average ratios of false positives for each tool and in total. It shows that on average, half of the positives from FindBugs are false and still nearly a third from PMD. QJ Pro has the worst result with only $4 \%$ of the positives being true positives. This leads to an overall average ratio of 0.66, which means that two thirds of the positives lead to unnecessary work. However, we have to notice that FindBugs and PMD are significantly better than that average. 
Table 2. Average ratios of false positives for each tool and in total

\begin{tabular}{|r|r|r|r|}
\hline FindBugs & PMD & QJ Pro & Total \\
\hline 0.47 & 0.31 & 0.96 & 0.66 \\
\hline
\end{tabular}

An illustrative example is the defect type "Return value of function is ignored". FindBugs only shows 4 warnings that all are true positives, whereas QJ Pro provides 689 further warnings that actually are not relevant. Because all the warnings have to be looked at, FindBugs is in this case much more efficient than the other two tools.

The efficiency of the tools varied over the projects. For the projects $B$ and $D$, the detection of the defect type "Database connection not closed" shows only warnings for true positives with FindBugs. For project $A$, it issued 46 warnings for which the database connection is actually closed. Similarly, the detection rate of true positives decreases for the projects $D$ and $A$ for the other two tools, with the exception of the well recognised positives from the maintainability category by PMD. This suggests that the efficiency of the defect detection depends on the design and the individual programming style, i.e. the implicit assumptions of the tool developers about how "good" code has to look like.

A recommendation of usage of the tools is difficult because of the issues described above. However, it suggests that QJ Pro, although it finds sometimes more defects than the other tools, has the highest noise ratio and therefore is the least efficient. FindBugs and PMD should be used in combination because the former finds many different defect types and the latter provides very accurate results in the maintainability category. Finally, PMD as well as QJ Pro can be used to enforce internal coding standards, which was ignored in our analysis above.

\subsection{Bug Finding Tools Versus Review}

An informal review was performed only on project $C$. The review team consisted of three developers, including the author of the code. The reviewers did not prepare specifically for the review but inspected the code at the review meeting.

Data. The review revealed 19 different types of defects which are summarised in Tab. 3 with their categories and number of occurrences.

Observations and Interpretations. All defects found by bug finding tools were also found by the review. However, the tools found 7 defects of type "Variable initialised but not used" in contrast to one defect revealed by the review. On the other hand, the review detected 8 defects of type "Unnecessary if-clause", whereas the tools only found one. The cause is that only in the one defect that was found by both there was no further computation after the if-clause. The redundancy of the others could only be found out by investigating the logics of the program. 
Table 3. Summary of the defect types and defects found by the review

\begin{tabular}{|l|r|r|}
\hline Defect Type & Category & Occurrences \\
\hline Database connection is not closed & 1 & 1 \\
Error message as return value & 1 & 12 \\
Further logical case ignored & 2 & 1 \\
Wrong result & 2 & 3 \\
Incomplete data on error & 2 & 3 \\
Wrong error handling & 2 & 6 \\
ResultSet is not closed & 4 & 1 \\
Statement is not closed & 4 & 1 \\
Difficult error handling & 4 & 10 \\
Database connection inside loop opened and closed & 4 & 1 \\
String concatenated inside loop with "+" & 4 & 1 \\
Unnecessary parameter on call & 5 & 51 \\
Unnecessary parameter on return & 5 & 21 \\
Complex for loop & 5 & 2 \\
Array initialised from 1 & 5 & 21 \\
Unnecessary if clauses & 5 & 8 \\
Variable initialised but not used & 5 & 1 \\
Complex variable increment & 5 & 1 \\
Complex type conversion & 5 & 7 \\
\hline
\end{tabular}

Apart from the two above, 17 additional types of defects were found, some of which could have been found by tools. For example, the concatenation of a string with "+" inside a loop is sometimes not shown by FindBugs although it is generally able to detect this defect type. Also, the defect that a database connection is not closed was not found, because this was done in different functions. Furthermore it was not discovered by the tools that the ResultSet and the corresponding Statement was never closed.

Other defect types such as logical faults or a wrong result from a function cannot be detected by bug finding tools. These defects, however, can be found during a review by following test cases through the code.

In summary, the review is more successful than bug finding tools, because it is able to detect far more defect types. However, it seems to be beneficial to first use a bug finding tool before inspecting the code, so that the defects that are found by both are already removed. This is because the automation makes it cheaper and more thorough than a manual review. However, we also notice a high number of false positives from all tools. This results in significant non-productive work for the developers that could in some cases exceed the improvement achieved by the automation.

\subsection{Bug Finding Tools Versus Testing}

We used black box as well as white box tests for system testing the software but also some unit tests were done. The black box tests were based on the textual specifications and the experience of the testers. Standard techniques 
Table 4. Summary of the defect types and defects found by the tests

\begin{tabular}{|l|r|r|}
\hline Defect Type & Category & Occurrences \\
\hline Data range not checked & 1 & 9 \\
Input not checked for special characters & 1 & 6 \\
Logical error on deletion & 1 & 1 \\
Consistency of input not checked & 2 & 3 \\
Leading zeros are not ignored & 2 & 1 \\
Incomplete deletion & 2 & 2 \\
Incomprehensible error message & 3 & 7 \\
Other logical errors & 2 & 3 \\
\hline
\end{tabular}

such as equivalence and boundary testing were used. The white box tests were developed using the source code and path testing. Overall several hundred test cases were developed and executed. A coverage tool has also been used to check the quality of the test suites. However, there were no stress tests which might have changed the results significantly. Only for the projects $E S t A$ and $C$, defects could be found. The other projects are probably too mature to be able to find further defects by normal system testing.

Data. The detected defect types together with their categories and the number of occurrences are summarised in Tab. 4. We also give some information on the coverage data that was reached by the tests. We measured class, method, and line coverage. The coverage was high apart from project $C$. In all the other projects, class coverage was nearly $100 \%$, method coverage was also in that area and line coverage lay between 60 and $93 \%$. The low coverage values for project $C$ might be explained by the fact that we invested the least amount of effort in testing this project.

Observations and Interpretations. The defects found by testing are in the categories Failure of the application, Logical failure, and Insufficient error handling. The analysis above of the defects showed that the bug finding tools predominantly find defects from the category Maintainability of the code. Therefore the dynamic test techniques find completely different defects.

For the software systems for which defects were revealed, there were no identical defects found with testing as well as the bug finding tools. Furthermore, the tools revealed several defects also in the systems for which the tests were not able to find one. These are defects that can only be found by extensive stress tests, such as database connections that are not closed. This can only result in performance problems or even a failure of the application, if the system is under a high usage rate and there is a huge amount of database connections that are not closed. The most defects, however, are really concerning maintainability and are therefore not detectable by dynamic testing.

In summary, the dynamic tests and the bug finding tools detect different defects. Dynamic testing is good at finding logical defects that are best visible 
Table 5. The defect removal efficiencies per defect-detection technique

\begin{tabular}{|l|r|r|}
\hline Technique & Number of Defects & Efficiency \\
\hline Bug Finding Tools & 585 & $76 \%$ \\
Review & 152 & $20 \%$ \\
Tests & 32 & $4 \%$ \\
\hline Total & 769 & $100 \%$ \\
\hline
\end{tabular}

Table 6. The defect removal efficiencies for each category

\begin{tabular}{|r|r|r|r|r|}
\hline Category & Bug Finding Tools & Reviews & Tests & Total \\
\hline 1 & $22 \%(8)$ & $35 \%(13)$ & $43 \%(16)$ & $100 \%(37)$ \\
2 & $15 \%(4)$ & $50 \%(13)$ & $35 \%(9)$ & $100 \%(26)$ \\
3 & $85 \%(40)$ & $0 \%(0)$ & $15 \%(7)$ & $100 \%(47)$ \\
4 & $70 \%(32)$ & $30 \%(14)$ & $0 \%(0)$ & $100 \%(46)$ \\
5 & $82 \%(501)$ & $18 \%(112)$ & $0 \%(0)$ & $100 \%(613)$ \\
\hline
\end{tabular}

when executing the software, bug finding tools have their strength at finding defects related to maintainability. Therefore, we again recommend using both techniques in a project.

\subsection{Defect Removal Efficiency}

The defect removal efficiency is as proposed by Jones in [1] the fraction of all defects that were detected by a specific defect-detection technique. The main problem with this metric is that the total number of defects cannot be known. In our case study we use the sum of all different defects detected by all techniques under consideration as an estimate for this number. The results are shown in Tab. 5. The metric suggests that the tools are the most efficient techniques whereas the tests where the least efficient.

However, we also have to take the defect categorisation into account because this changes the picture significantly. The Tab. 6] shows the efficiencies for each techniques and category with the number of defects in brackets. It makes obvious that tests and reviews are far more efficient in finding defects of the categories 1 and 2 than the bug finding tools which are the most severe defects.

\section{Discussion}

The result that bug finding tools mainly detect defects that are related to the maintainability of the code complies with the expectation an experienced developer would have. Static analysis only allows to look for certain patterns in the code and simple dataflow and controlflow properties. Therefore only reviews or tests are able to verify the logic of the software (as long as the static analysis is not linked with model checking techniques). The tools do not "understand" 
the code in that sense. The prime example for this is the varying efficiency over the projects. In many cases, the tools were not capable to realise that certain database connections are not closed in the same Java method but a different one. They only search for a certain pattern. Therefore, the limitation of static analysis tools lies in what is expressible by bug patterns, or in how good and generic the patterns can be.

However, it still is surprising that there is not a single overlapping defect detected by bug finding tools and dynamic tests. On the positive side, this implies that the two techniques are perfectly complementary and can be used together with great benefit. The negative side is that by using the automated static analysis techniques we considered, it may not be possible to reduce costly testing efforts. That there is only little overlapping follows from the observation above that the tools mainly find maintenance-related defects. However, one would expect to see at least some defects that the tests found also detected by the tools, especially concerning dataflow and controlflow. The negative results in this study can be explained with the fact that most of the projects analysed are quite mature, and some of them are already in operation. This resulted in only a small number of defects that were found during testing which in turn could be a reason for the lack of overlapping.

A rather disillusioning result is the high ratio of false positives that are issued by the tools. The expected benefit of the automation using such tools lies in the hope that less human intervention is necessary to detect defects. However, as on average two thirds of the warnings are false positives, the human effort could be even higher when using bug finding tools because each warning has to be checked to decide on the relevance of the warning. Nevertheless, there are significant differences between the tools so that choosing the best combination of tools could still pay off.

Bug finding tools that use additional annotations in the code for defectdetection could be beneficial considering the overlap of defects with other techniques as well as the false positives ratio. The annotations allow the tool to understand the code to a certain extent and therefore permits some checks of the logic. This deeper knowledge of the code might reduce the false positives ratio. However, to make the annotations requires additional effort by the developers. It needs to be analysed if this effort is lucrative.

The effort and corresponding costs of the determination of defects using the tools (including checking the false positives) was not determined in this study. This is however necessary to find out if the use of bug finding tools is beneficial at all.

\section{Related Work}

There are only few studies about how bug finding tools relate among themselves and to other established defect-detection techniques such as testing or reviews.

In [18] among others PMD and FindBugs are compared based on their warnings which were not all checked for false positives. The findings are that although 
there is some overlap the warnings generated by the tools are mostly distinct. We can support this result with our data.

Engler and Musuvathi discuss in 6] the comparison of their bug finding tool with model checking techniques. They argument that static analysis is able to check larger amounts of code and find more defects but model checking can check the implications of the code not just properties that are on the surface.

In [10] a static analysis tools for $\mathrm{C}$ code is discussed. The authors state that sophisticated analysis of, for example, pointers leads to far less false positives than simple syntactical checks.

An interesting combination of static analysis tools and testing in described in 5. It is proposed to use static analysis to find potential problems and automatically generate test cases to verify if there is a real defect. However, the approach obviously does not work with maintenance-related defects.

Bush et al. report in [3] on a static analyser for $\mathrm{C}$ and $\mathrm{C}++$ code which is able to find several more dynamic programming errors. However, a comparison with tests was not done. Nevertheless, our observation that the defect-finding capabilities depend strongly on the coding styles of different programmers is supported in this paper.

In 22] an evaluation of static analysis tools for $\mathrm{C}$ code regarding buffer overflows is described. The defects were injected and the fraction of buffer overflows found by each technique was measured. It is also noted that the rates of false positives or false alarms are unacceptably high.

Palsberg describes in [14] some bug finding tools that use type-based analysis. He shows that they are able to detect race conditions or memory leaks in programs.

\section{Conclusion}

The work presented is not a comprehensive empirical study but a case study using a series of projects mainly from an industrial environment giving first indications of how the defects found by bug finding tools relate to other defectdetection techniques.

The main findings are that the bug finding tools revealed completely different defects than the dynamic tests but a subset of the types of the review. The defect types that are detected by the tools are analysed more thoroughly than with reviews. The effectiveness of the tools seems to strongly depend on the personal programming style and the design of the software as the results differed strongly from project to project. Finally, a combination of the usage of bug finding tools together with reviews and tests would be most advisable if the number of false positives were lower. It probably costs more time to resolve the false positives than is saved by the automation using the tools.

Therefore, the main conclusion is that bug finding tools can save costs when used together with other defect-detection techniques, if the tool developers are able to improve the tools in terms of the false positives ratio and tolerance of different programming styles. 


\section{$9 \quad$ Future Work}

This study is only a first indication and needs further empirical validation to be able to derive solid conclusions. For this, we plan to repeat this study on different subjects and also taking other tools into account, e.g. commercial tools or tools that use additional annotations in the source code. Also, the investigation of other types of software is important, since we only considered web applications in this study.

How the proper combination of the different techniques can be found is also subject to further research. As a first step more reliability-oriented measures, such as the failure intensity efficiency [19,20] can be used to compare the bug finding tools with other techniques. This can give more clues in terms of the effect on the reliability of the usage of bug finding tools. However, a comprehensive treatment of the subject needs to incorporate the false positives ratio into a cost model based on 21] to be able to determine the economically best alternatives.

\section{Acknowledgments}

We want to thank the authors of the tools FindBugs, PMD, QJ Pro for investing such an amount of work in the tools and making them available to the public.

\section{References}

1. T. Ball and S.K. Rajamani. The SLAM Project: Debugging System Software via Static Analysis. In Proc. 29th Annual ACM SIGPLAN-SIGACT Symposium on Principles of Programming Languages, 2002.

2. B. Beizer. Software Testing Techniques. Thomson Learning, 2nd edition, 1990.

3. W.R. Bush, J.D. Pincus, and D.J. Sielaff. A static analyzer for finding dynamic programming errors. Softw. Pract. Exper., 30:775-802, 2000.

4. R. Chillarege. Orthogonal Defect Classification. In Michael R. Lyu, editor, Handbook of Software Reliability Engineering, chapter 9. IEEE Computer Society Press and McGraw-Hill, 1996.

5. C. Csallner and Y. Smaragdakis. CnC: Combining Static Checking and Testing. In Proc. 27th International Conference on Software Engineering (ICSE'05), 2005. To appear.

6. D. Engler and M. Musuvathi. Static Analysis versus Model Checking for Bug Finding. In Proc. Verification, Model Checking and Abstract Interpretation (VMCAI'04), volume 2937 of LNCS, pages 191-210. Springer, 2002.

7. C. Flanagan, K.R.M. Leino, M. Lillibridge, G. Nelson, J.B. Saxe, and R. Stata. Extended Static Checking for Java. In Proc. 2002 ACM SIGPLAN Conference on Programming Language Design and Implementation, 2002.

8. D. Hovemeyer and W. Pugh. Finding Bugs is Easy. SIGPLAN Notices, 39(12), 2004. To appear.

9. IEEE. IEEE Standard Classification for Software Anomalies, 1993. IEEE Std 1044-1993.

10. R. Johnson and D. Wagner. Finding User/Kernel Pointer Bugs With Type Inference. In Proc. 13th USENIX Security Symposium, 2004. 
11. C. Jones. Applied Software Measurement: Assuring Productivity and Quality. McGraw-Hill, 1991.

12. C. Koller. Vergleich verschiedener Methoden zur analytischen Qualitätssicherung. Diploma Thesis, Technische Universität München, 2004. In German.

13. G.J. Myers. The Art of Software Testing. John Wiley \& Sons, 1979.

14. J. Palsberg. Type-Based Analysis and Applications. In Proc. 2001 ACM SIGPLAN-SIGSOFT Workshop on Program Analysis for Software Tools and Engineering (PASTE'01), pages 20-27. ACM Press, 2001.

15. PMD. http://pmd.sourceforge.net (February 2005).

16. A. Pretschner, W. Prenninger, S. Wagner, C. Kühnel, M. Baumgartner, B. Sostawa, R. Zölch, and T. Stauner. One Evaluation of Model-Based Testing and its Automation. In Proc. 27th International Conference on Software Engineering (ICSE'05), 2005. To appear.

17. QJ Pro. http://qjpro.sourceforge.net (February 2005).

18. N. Rutar, C.B. Almazan, and J.S. Foster. A Comparison of Bug Finding Tools for Java. In Proc. 15th IEEE International Symposium on Software Reliability Engineering (ISSRE'04), pages 245-256, 2004.

19. S. Wagner. Efficiency Analysis of Defect-Detection Techniques. Technical Report TUMI-0413, Institut für Informatik, Technische Universität München, 2004.

20. S. Wagner. Reliability Efficiency of Defect-Detection Techniques: A Field Study. In Suppl. Proc. 15th IEEE International Symposium on Software Reliability Engineering (ISSRE'04), pages 294-301, 2004.

21. S. Wagner. Towards Software Quality Economics for Defect-Detection Techniques. In Proc. 29th Annual IEEE/NASA Software Engineering Workshop, 2005. To appear.

22. M. Zitser, R. Lippmann, and T .Leek. Testing Static Analysis Tools using Exploitable Buffer Overflows from Open Source Code. In Proc. 12th ACM SIGSOFT International Symposium on Foundations of Software Engineering (SIGSOFT'04/FSE-12), pages 97-106. ACM Press, 2004. 